\title{
L'angoisse de l'écriture dans l'œuvre de Germaine Guèvremont
}

\author{
Andréa KING \\ Université Queen's
}

L'écriture est un refuge pour Germaine Guèvremont. Dans une interview parue en 1959, elle insiste sur le fait qu'elle a commencé à écrire "pour [s]'évader de l'inquiétude et de la peur » (Bouthillette, 1959, p. 7). Cette peur - à en croire la paraphrase fournie par l'intervieweur - serait née de l'éclatement des valeurs traditionnelles paysannes, lesquelles ne répondaient plus à la réalité sociale et économique. Or, l'«évasion " de la peur dont parle Guèvremont n'en est pas vraiment une, car écrire en tant que femme à son époque voulait dire bouleverser ces mêmes valeurs traditionnelles et, par conséquent, faire face à la peur. 
La crainte de contribuer à l'ébranlement d'une ancienne tradition n'est certainement pas unique aux écrivaines québécoises des années 1940. Dans The Madwoman in the Attic (1979), les théoriciennes féministes américaines Susan Gilbert et Susan Gubar montrent que, très souvent, la crainte et l'ambivalence marquent l'expérience de celles qui s'engagent à écrire dans une société patriarcale dépourvue d'une solide tradition littéraire au féminin ${ }^{1}$, comme c'était le cas pour Guèvremont. Dans un tel contexte, l'écrivaine occupe une position de solitude symbolique; ainsi fait-elle preuve de courage et de compétence, mais non sans éprouver de l'angoisse, car elle tente de jouer un rôle autre que celui qui lui est prescrit.

Créer et s'isoler? Ou s'effacer et se conformer? Voilà l'impasse qui donne lieu à ce que Gilbert et Gubar appellent «l'angoisse de l'écriture» (anxiety of authorship) : la crainte éprouvée par l'écrivaine que sa création l'isole et, finalement, la détruise. Gilbert et Gubar décrivent cette angoisse comme " [an] isolation that felt like illness, an alienation that felt like madness» (p. 51). L'écrivaine qui la met en scène place ses personnages féminins dans des rôles conventionnels, mais construit aussi pour eux des portes de sortie psychologiques:

\footnotetext{
${ }^{1}$ Les théories de Gilbert et Gubar ont fait l'objet de critiques rigoureuses de la part de certaines féministes. On désapprouve leur tendance à réunir toutes les écrivaines sous la même théorie (peu importe leur milieu socioculturel), et à insinuer que le texte littéraire constitue l'assemblage des désirs inconscients de l'écrivaine. Il n'en demeure pas moins que ces théories élucident la forte présence de certaines maladies dans des textes écrits par des femmes vivant dans une société patriarcale, aussi bien que la raison pour laquelle ces maladies n'atteignent que les personnages féminins. Sans prétendre résoudre ce débat, je lirai l'œuvre de Guèvremont comme étant symptomatique d'une tension culturelle et esthétique qui est autant occidentale que québécoise.
} 
since they were trapped in so many ways in the architecture both the houses and the institutions - of patriarchy, women expressed their anxiety of authorship by comparing their «presumptuous» literary ambitions with the domestic accomplishments that had been prescribed for them. Inevitably, too, they expressed their claustrophobic rage by enacting rebellious escapes. (p. 85)

Ces «fuites rebelles » ne sont cependant pas héroïques, mais plutôt maladives: le suicide, la folie, l'anorexie et la boulimie (qui, toutes deux, mènent à l'évasion par excellence qu'est la mort) figurent parmi elles.

Les maladies nommées ci-dessus sont justement mises en évidence dans l'œuvre de Guèvremont, comme on le verra dans l'analyse qui suit, et qui portera non seulement sur le cycle du Survenant mais aussi sur le roman-feuilleton Tu seras journaliste ${ }^{2}$, publié dans la revue Paysana entre 1939 et 1940. Bien que Phonsine soit la figure privilégiée de cette lecture, il sera également question d'autres personnages féminins qui forment une lignée de femmes «traditionnelles» mais maladives, dont plusieurs témoignent d'une créativité refoulée. L'analyse de ces personnages me permettra de montrer que l'écriture au féminin était encore un acte ambivalent à l'époque où Guèvremont a rédigé les quatre œuvres en question.

La deuxième partie de l'article examinera la figure de la revenante, comprise ici non seulement dans son sens traditionnel (l'apparition spectrale du personnage féminin mort), mais aussi dans un sens plus large, à savoir le

\footnotetext{
2 Notons que cet ouvrage n'est pas de la même qualité littéraire que le cycle du Survenant, et plus particulièrement du diptyque du Survenant et de MarieDidace (une critique constate qu'il a « la valeur d'un honnête devoir d'écolier » (Leclerc, 1963, p. 33)). L'intérêt de Tu seras journaliste provient de son caractère autobiographique et des échos annonciateurs du Cycle.
} 
redoublement de la femme morte ${ }^{3}$, ou bien la mise en scène du cadavre de celle-ci. La figure de la revenante participe de l'angoisse de l'écriture dans la mesure où elle personnifie la tension entre la création et l'effacement du féminin : en elle, ces deux possibilités se juxtaposent et se superposent.

\section{Une bonne maladie}

Phonsine, dans Marie-Didace, fait un rêve récurrent inquiétant : elle, sa tasse préférée et sa petite fille tombent dans le puits des Beauchemin (p. 177)4. Lorsqu'elle consulte le médecin sur ce rêve, il lui répond : "Sais-tu ce qu'il te faudrait? Quelque bonne maladie qui t'empêcherait de penser. Ça te nettoyerait les idées avec le sang » (p. 177). À en croire le docteur, Phonsine, pour soigner son esprit inquiet, n'aurait qu'à tomber malade: « nettoyer les idées avec le sang. »

Comme l'ont montré de nombreuses théoriciennes féministes (Bordo, 1987; Battersby 1989; Grosz, 1994), le «sang» - à savoir tout ce qui est associé au corps - a longtemps été le domaine des femmes. Les hommes, épargnés par les menstruations et l'accouchement, étaient au contraire associés au domaine de la pensée, de la philosophie, de la créativité. La conversation entre Phonsine et le docteur met en relief une aporie fascinante : d'une part, les paroles du médecin infantilisent Phonsine, minimisent ses inquiétudes légitimes et

\footnotetext{
${ }^{3}$ Comme le souligne Elisabeth Bronfen, la figure du double de la femme morte joue le rôle de revenante, car " [s] he is denied her own body, and is only a figure for a meaning alterior to herself, prematurely turned into a ghost [...] She simulates a dead woman and in so doing signifies both her own division and her social non-existence » (p. 328).

${ }^{4}$ Dorénavant, Marie-Didace sera identifiée par le sigle MD et Le Survenant par LS.
} 
l'invitent à sombrer plus profondément dans la maladie; d'autre part, la représentation de cet échange et du mal-être du personnage féminin fonctionne comme une critique de ce même discours. L'échange entre Phonsine et le médecin évoque la vieille opposition corps/esprit, opposition qui a longtemps empêché la participation des femmes aux activités intellectuelles.

Phonsine représente un exemple de créativité refoulée, ce qui est mis en scène dans le passage du Survenant où elle songe nostalgiquement au travail à l'aiguille auquel elle s'adonnait autrefois : "croyant les hommes aux champs» (p. 49), elle manipule une pointe de velours et se rappelle le plaisir qu'elle éprouvait «à imaginer les porte-balais, les pelotes à épingles et tous les beaux objets qu'elle pourrait façonner de ses mains et enfouir dans un tiroir dans du papier de soie » (p. 48). Tout en rêvant, cependant, elle s'interdit « des pensées frivoles de la sorte » (p. 49). Le lecteur entend derrière cette censure la voix désapprobatrice du père Didace, qui, au début du Survenant, la décrit comme « [u]ne femme qui ne pèse pas le poids [...], sans même un petit dans les bras, après trois années de ménage » ( $\mathrm{p}$. 29). La seule personne qui encourage le penchant créatif de Phonsine est le Survenant, qui lui donne en cadeau une brassée de foin d'odeur qui servira à ses « petits ouvrages » (LS, p. 49).

L'ambivalence qu'éprouve Phonsine devant les limitations de son rôle de femme Beauchemin paraît aussi dans son attitude envers la grossesse. Lorsqu'elle tombe enceinte à la fin du Survenant, elle est consciente que le fait de porter un enfant fera d'elle une "vraie » Beauchemin, un statut qui lui a été refusé jusqu'alors : "Quand elle aurait donné à la famille un Didace de plus, elle saurait bien prendre sa place dans la belle 
confrérie des dames Beauchemin »(LS, p. 200). Dans MarieDidace, toutefois, elle fait tout pour cacher son état jusqu'au moment où son mari Amable le révèle dans un mouvement de colère contre l'Acayenne. Phonsine aspire à prendre la place de Mathilde Beauchemin (la mère par excellence) après la mort de cette dernière, mais il est vrai qu'il s'agit d'un rôle pour le moins accablant: en effet, son mari la décrit comme une "sainte femme, qui n'avait rien à soi, qui pouvait s'arracher le cœur pour combler les autres » (MD, p. 111). Ailleurs le père Didace se souvient de «la vraie femme qu'il avait! » tout en faisant un dur constat : « elle était morte, usée de peine » (LS, p. 197).

Alors que Phonsine, par ses paroles, professe une admiration pour Mathilde (et pour sa fille Marie-Amanda) et jalouse l'Acayenne (qui est aussi meilleure ménagère), ses actions montrent qu'elle tente de repousser la réalité de la maternité ainsi que le rôle de mère qui la menace. Maintes références à sa maigreur et à son habitude de ne presque pas manger - six occurrences dans Le Survenant, et le triple dans Marie-Didacepermettent de considérer Phonsine comme anorexique. Elle mange à peine durant sa grossesse, état qui ne sera connu qu'une semaine avant l'accouchement ${ }^{5}$, ce qui lui donne de la satisfaction : «s'efforçant de paraître encore plus maigre, [elle] abaissa lentement la vue sur son ventre. Non, personne ne pouvait deviner son état de grossesse » (MD, p. 103).

La nature publique de la fin de sa grossesse l'affole : «Son front, puis le haut de ses joues prirent le masque de la

\footnotetext{
5 Le commentaire de Phonsine qu'elle «[a] encore cinq grosses semaines à attendre » (Guèvremont, 1992, p. 121) est fait le même jour où Amable révèle la grossesse de sa femme à Didace et à l'Acayenne. Ensuite Marie-Didace naît prématurément à huit mois, soit une semaine plus tard.
} 
grossesse. Elle mangeait à peine. Et de savoir son état l'objet des conversations, des discussions et des calculs, elle se sentit frustrée en sa personne, et plus abandonnée » (MD, p. 127). Elle se nourrit si mal que non seulement elle n'arrive pas à effectuer les travaux ménagers que le père Didace attend d'elle, mais qu'il est aussi permis de se demander si elle ne cherche pas à provoquer une fausse couche. Sa décision, à presque huit mois de grossesse, de marcher de Sorel jusqu'à Sainte-Anne sans avoir mangé renforce l'hypothèse d'un rejet inconscient de la maternité; ce surmenage provoquera la naissance prématurée de Marie-Didace, et risquera de coûter la vie à la mère et à l'enfant. Après l'accouchement, Phonsine mange si peu qu'elle ne parvient même pas à allaiter son enfant (MD, p. 177); l'odeur du lait chaud la dégoûte, d'ailleurs, depuis le début de sa grossesse (MD, p. 38).

Son corps faisant défaut, Phonsine ne peut donc pas remplir sa fonction de mère, et c'est l'Acayenne qui jouera ce rôle auprès de l'enfant, qui préférera d'ailleurs le sein de la belle-mère à celui de Phonsine: "Maman: un corsage noir et dur, où sa petite tête se heurte à chercher en vain un coin propice au sommeil. Me-mère : un vaste corsage fleuri, moelleux et chaud, qui se soulève en grandes vagues et en chansons. » (MD, p. 160)

Non seulement le corps de Phonsine est-il devenu le site privilégié d'une tension entre l'acceptation des structures patriarcales qui l'enferment-celles que Patricia Smart a appelées « la Maison du Père » (1988) ${ }^{6}$, , et le rejet violent de celles-ci, mais son corps est cette maison, l'abri d'« un Didace de

\footnotetext{
${ }^{6}$ Selon Smart, " [é]crire est bien une activité qui se poursuit dans la Maison du Père; cette maison étant évidemment une métaphore de la culture et de ses structures de représentation idéologiques, artistiques et langagières, dont nous comprenons de plus en plus clairement depuis l'émergence du féminisme qu'elles sont la projection d'une subjectivité et d'une autorité masculines » (1988, p. 22).
} 
plus ». Or, pour Phonsine, miner le corps veut dire défaire la maison. Les implications de cet affaiblissement corporel sont encore plus importantes si l'on se rappelle que la grossesse est une métaphore à laquelle de nombreux écrivains - hommes et femmes - ont recours pour représenter l'écriture. L'ambivalence de Phonsine face à sa grossesse serait-elle une métaphore de la "peur» dont parle Guèvremont lorsqu'elle décrit les difficultés d'écrire à son époque? Et qui sont les autres personnages féminins qui prennent part à cette même représentation équivoque?

\section{Figures du double}

Gilbert et Gubar soutiennent que l'écrivaine qui cherche à représenter mimétiquement les obstacles à la créativité au féminin s'engage dans la duplicité, car elle se sert d'un modèle esthétique et social masculin et crée un objet qui ne la reflète pas, les personnages féminins ne réussissant pas à se libérer de l'univers patriarcal, alors que l'écrivaine y parvient, sans quoi son texte n'existerait pas (1979, p. 69). Ce type d'écriture implique une abnégation importante :

[...] though writers [...] do use masks and disguises in most of their work, though what Keats called 'the poetical character' in some sense has 'no self' because it is so many selves, the continual use of male models inevitably involves the female artist in a dangerous form of psychological self-denial that goes far beyond the metaphysical self-lessness Keats was contemplating. (Gilbert et Gubar, 1979, p. 69)

Comme si le texte mimait son contexte de création, le paradoxe « abnégation/création » est mis en relief dans l'œuvre de Guèvremont non seulement par la corporalité problématique 
de Phonsine et son rapport à la création, mais aussi par le redoublement du personnage féminin malheureux et maladif.

Considérons par exemple le personnage de l'Acayenne. David Décarie (2001 et 2006) voit en elle un double du Survenant, mais on peut aussi se demander si elle ne serait pas le double inversé de Phonsine. De façon significative, elle est la première à deviner que la bru est enceinte (MD, p. 114), et tout au long de Marie-Didace, Phonsine maigrit tandis que l'Acayenne grossit?. À la fin du roman, Phonsine "pèse moins qu'une plume» (p. 218), alors que l'Acayenne, qui «n'est pas grasse de rien », comme le remarque le docteur (p. 208), mange toute une assiettée de ragoût de porc quelques heures avant sa mort contre les conseils de celui-ci. Phonsine s'efforce de maîtriser son corps, ce qu'elle réussit à faire jusqu'à son accouchement, où, à son plus grand désarroi, «[s]on corps moulu n'obéi[t] plus à sa volonté » (p. 144); tout à l'opposé, l'Acayenne déborde. Le passage décrivant le retour de Phonsine de Sorel met en évidence la relation contraire qu'ont ces deux femmes avec leur corps. Phonsine est « pitoyable près du poêle, un petit châle serré sur sa poitrines, la déformation de son corps accentuant la maigreur de ses épaules et de sa figure », tandis que l'Acayenne, quant à elle, «se ber[ce], en mangeant une pomme dont le jus coul[e] sur son menton. » (p. 142)

Les antithèses abondent: Phonsine est faible, l'Acayenne est forte et travailleuse; Phonsine est associée au puits espace étroit et clos - alors que l'Acayenne est liée à la mer, qui, elle, est vaste, fluide et mouvante. L'Acayenne représente

\footnotetext{
7 Le charretier qui conduit le juge et l'avocat jusqu'à la maison des Beauchemin remarque ce changement chez l'Acayenne (Guèvremont, 1992, p. 125).

${ }^{8}$ Notons bien le « $\mathrm{p}$ » allitératif ici, qui met en relief l'adjectif « pitoyable ».
} 
l'envers de Phonsine, mais sans échapper totalement aux contraintes qui rendent l'autre malade, étant victime de son ambivalence. Elle est peut-être une « grosse mangeuse » (MD, p. 208), mais elle ne semble pas capable de réelles jouissances. Elle se caractérise par sa nostalgie pour son ancienne vie et son mari décédé, son apathie face au père Didace et au Chenal du Moine, et même de la jalousie à l'égard de sa belle-fille. Qui plus est, à la veille de sa mort, elle est représentée en proie à une douleur qui rappelle la malheureuse Phonsine: «Les mains jointes lâchement entre ses genoux, l'Acayenne était assise, pliée en deux, près du poêle » (MD, p. 209). Les deux femmes se définissent par le manque, la mélancolie. À la fin du roman, Phonsine devient folle; l'Acayenne avale une boîte de pilules dans la nuit et en meurt. Si le suicide n'est pas explicite dans cette scène, il est du moins fortement suggéré et appuyé par la prégnance de ce thème dans d'autres textes de Guèvremont.

Ce redoublement des personnages féminins mélanco-liques peut se lire comme une critique sévère de l'ordre social patriarcal, mais cela ne va pas sans quelque ambiguïté, comme en témoigne la correspondance de Guèvremont avec le poète Alfred DesRochers. Entre le 16 septembre 1942 et le 15 janvier 1951 - époque durant laquelle Guèvremont rédigeait Le Survenant et MarieDidace - l'écrivaine écrivit 111 lettres à son ami et mentor. Ces lettres sont encore inédites, mais certains passages concernant Marie-Didace ont été publiés dans un article d'Yvan Lepage (1990). Celui-ci constate que la plupart des lettres portent la formule d'appel « Cher Survenant » ou bien « Cher père Didace » et sont signées « Marie-Amanda » (p. 66).

Guèvremont cherchait donc des conseils auprès de l'homme qui lui servait de modèle pour le Survenant, homme 
viril et nomade, et pour son patriarche, le père Didace'. Il y a ici un paradoxe fascinant dans la mesure où Guèvremont sollicitait l'avis du patriarche qu'elle essayait symboliquement d'assassiner. En effet, à la fin de Marie-Didace, le père Didace meurt d'une crise cardiaque et le roman laisse croire que le Survenant est mort en " glorieux disparu » à la guerre. Encore plus problématique est le fait que Guèvremont s'approprie le nom de «Marie-Amanda», le personnage féminin qui correspond le mieux à l'idéal féminin dans l'ordre patriarcal. Toutefois, Guèvremont écrit ce qui suit à DesRochers lorsqu'elle lui envoie le premier chapitre de son deuxième roman :

Voici Marie-Didace avec mes craintes et mes espoirs. Et même si je voudrais vous dire le contraire, je vous demande de ne ménager ni les uns, ni les autres. Mon mari l'aime. J'attends votre jugement. Puisque c'est le point de départ de toute une envolée. La crainte de Phonsine s'y dessine. Elle ne fera que s'accentuer. (Lepage, 1990, p. 68-69, je souligne)

La correspondance de l'auteure laisse donc transparaître une identification avec le personnage plus trouble de Phonsine.

Il faut préciser que l'œuvre de Guèvremont laisse entendre un désir de faire sortir la femme du rôle effacé qui lui a été réservé par la tradition littéraire et sociale. D'autres critiques ont exploré les aspects transgressifs du cycle du Survenant. Smart, par exemple, en souligne l'ouverture vers l'autre, la valorisation de la jouissance corporelle et l'évocation d'une relation de non-possession $\left(1988\right.$, p. 137-166) ${ }^{10}$. Lori

9 On peut se demander si Guèvremont, en appelant DesRochers «père Didace», brouille les pistes d'un rapport amoureux qui aurait été trop transparent avec la seule identification au Survenant.

10 Il faut remarquer que Smart nuance sa position par rapport à la nature subversive de l'œuvre de Guèvremont dans un article plus récent où elle maintient que, «en général, [Guèvremont], tout en nous donnant à voir 
Saint-Martin, pour sa part, examine la remise en cause du rôle des sexes à travers le dialogue des personnages féminins (1997). Il n'en demeure pas moins que ce qui frappe à la fin de Marie-Didace, c'est l'angoisse de Phonsine face au rôle de mère dont elle hérite une fois de plus, cette fois par rapport à l'Acayenne. Le passage où la bru masse le dos de l'Acayenne la veille de la mort de celle-ci met en scène ce transfert symbolique:

Une grimace de répugnance sur la figure, Phonsine retraita d'un pas. [...]

Mais mue par une impulsion plus forte que sa volonté, [Phonsine] appliqua [ses mains] subitement au dos de l'Acayenne. L'autre échappa un cri de surprise. Au contact de la peau étrangère, les longs doigts gauches refusèrent d'obéir; ils s'immobilisèrent, impuissants.

[...]

Les yeux fermés, la jeune femme, frémissante de dédain, recommença à frotter le dos nu. Elle frictionnait à sa force la peau que l'âge avait épaissie. Le grain en était lâche, et la graisse molle, sans résistance, formait de petites boursouflures. Une odeur fade s'en dégagea. (p. 211)

Phonsine tentera d'expulser d'elle-même ce rôle en vomissant à la fin du passage. Mais c'est en vain : l'Acayenne meurt cette même nuit et l'«odeur fade» de la mort colle désormais à Phonsine. Au lieu d'assumer son rôle de maîtresse de maison, qu'elle souhaite depuis si longtemps, Phonsine commence sa descente vers la culpabilité et la folie :

Des mains monstrueuses happèrent Phonsine; elles l'entraînèrent dans une cavalcade infernale que menait l'Acayenne, escortée de Pierre-Côme Provençal. Angélina galopait à côté en riant comme une folle. [...] Des quatre coins de la paroisse, les gens, à la face de

l'impasse des rôles féminins traditionnels, n'arriv[e] pas à envisager de possibilités nouvelles pour la femme » (1997, p. 18). 
démon, accouraient, fourche en main, pour l'entraîner en enfer, pendant l'éternité. (p. 213-214)

Ces «mains monstrueuses» qui saisissent Phonsine rappellent irrésistiblement les mains qui l'ont attaquée lorsqu'elle accouchait de Marie-Didace: «Des mains de feu la pétrissaient, la poussaient, l'entraînaient; elles l'abandonnèrent, solitaire, dans la rouge vallée de la maternité. Un cri résonna à travers la maison: le mystère commençait» (MD, p. 144). L'enfer qui enveloppe Phonsine après la mort de l'Acayenne ne se rapproche-t-il pas de cette « rouge vallée de la maternité »? Quant au cri qu'elle a lâché, il sera le seul; hormis cet exemple, Phonsine s'oblige au silence ${ }^{11}$, et lorsqu'elle sombre finalement dans la folie, elle le fait "sans un cri » (MD, p. 214). Il est significatif que dans la dernière vision infernale de Phonsine citée ci-dessus, ce soit au tour d'Angélina - qui prendra soin désormais de la petite Marie-Didace - de rire « comme une folle », ce qui n'est pas très éloigné, en fin de compte, du fait de crier devant la « rouge vallée de la maternité ».

La scène suivante offre un tableau de domesticité tranquille. Angélina cuisine chez elle, épluchant des pommes. Près du poêle, une chatte surveille son chaton qui joue à attraper les pelures. Ce rapprochement entre la folie infernale et la domesticité est-il révélateur? Certes, Angélina est le personnage féminin qui bouleverse le plus les valeurs traditionnelles explorées dans le cycle du Survenant. Elle insiste pour choisir son mari selon ses propres critères, par exemple, restant finalement célibataire après le départ du Survenant. Toutefois, il faut se rappeler qu'Angélina, ayant éprouvé un

11 L'Acayenne réprimande d'ailleurs Phonsine d'avoir crié au début de l'accouchement (Guèvremont, 1992, p. 144); le cri ne se renouvellera pas. 
«éblouissement intérieur» inspiré de la «musique» de certains passages poétiques dans son missel (LS, p. 55), avait espéré d'abord devenir sacristine, une vocation à ses yeux à la fois créative et passionnante. Ce fut le curé qui l'en dissuada: « il la trouva débile et bien jeune. Puis son infirmité lui serait un obstacle. Il l'engagea à prier et à attendre quelques années : une bonne enfant ne doit-elle pas en premier lieu assister son père, seul et dans le besoin? » (LS, p. 56). Angélina, bien entendu, «refoula son rêve» pour l'amour du père. Le personnage féminin le plus transgressif à l'égard du rôle des sexes fournit donc un autre exemple de la créativité au féminin entravée. Cet étouffement est d'ailleurs mis en relief et contesté par le déploiement de la spectralité.

\section{La revenante}

$\mathrm{Si}$, dans l'œuvre de Guèvremont, la présence de revenants comme Amable et le Survenant est manifeste ${ }^{12}$, celle des revenantes est beaucoup plus subtile. Le symbolisme de ces revenantes est néanmoins hautement significatif, car elles participent d'une tradition littéraire occidentale qui est bâtie sur l'effacement du féminin. Comme le montre Elisabeth Bronfen dans Over Her Dead Body: Death, Femininity and the Aesthetic,

[t]he cultural topos or image [...] presupposes and confirms that Woman is constructed as Other than man; as that which is not the centre of a social or representational system. Hers is a position of non-coherence, of the void or an empty space

12 Phonsine voit Amable dans un rêve peu après son accouchement (Guèvremont, 1992, p. 154); le Survenant redouble Ephrem, le fils Beauchemin noyé, dans la mesure où il témoigne d'une force et d'une vaillance analogues aux yeux de Didace. 
between signifiers precisely because she is constructed as the vanishing point and the condition of western culture's fictions of itself. (1992, p. 403)

Il n'y a pas de "position féminine » dans ce système de représentation, ce qui veut dire que lorsque l'écrivaine s'en empare, elle s'engage à l'effacement - voire à la mort : "[the woman writer] must not only assume a masculine position but she must also perform the position of absence, of the double, which is the rhetoric of death " (Bronfen, 1992, p. 406). Se tenir hors de ce système de représentation est impossible ${ }^{13}$, mais tout en acceptant de s'y laisser entraîner, l'écrivaine peut exposer la violence qui y est normalement occultée. Or, en déployant un topos comme la revenance, qui met en scène la disparition et la réapparition de la femme, l'écrivaine se place, inéluctablement, quelque part entre la complicité et la critique. Qui plus est, lorsque ce même topos est lié à la question de la créativité et de la crainte au féminin, comme dans le cas de Guèvremont, il attire justement l'attention sur la position difficile qu'occupe l'écrivaine: l'(in)existence de la revenante dévoile le (non-)lieu de l'auteure.

Le cas le plus exemplaire de la figure de la revenante chez Guèvremont est le personnage adolescent de Marie-Ange Defroi (surnommée «l'Ange à Defroi ») d'En pleine terre. "Cadeau » d'une mère agonisante à son mari, Marie-Ange naît dans la mort, vouée non seulement au rôle de remplaçante de la femme disparue, mais aussi à celui du double du père: «Mais sa

13 Pour Bronfen, l'écriture féministe n'échappe pas à cette impasse : "Onecould speculate whether feminist writing, aimed at denying complicity with culture by drafting a feminine subject position outside its alienation, does not merely miss the point that all human beings are irrevocably subjected by cultural language. More crucially this may be a strategy by which death once again [...] is repressed » (1992, p. 406). 
femme, en mourant, lui avait donné une fille pareille à lui » (p. 69). Cette fille, «pareille à lui », représente davantage l'altérité du père, car elle porte la mort (altérité par excellence) aussi bien dans son prénom («Ange») que dans son nom («Defroi »/« de froid»). La désignation «l'Ange à Defroi » met l'accent sur le possesseur masculin (le père), rappelant que la mort est le destin d'une fille désobéissante qui n'accepte pas son rôle comme simple miroir ou objet du père. En effet, l'adolescente attrape sa mystérieuse maladie en se rendant dans les marais pour y chasser la grenouille, ce qu'elle fait à l'encontre des consignes du père, et dans le but de s'acheter de beaux vêtements qui plairaient à un garçon qu'elle aime. L'histoire de l'Ange à Defroi est d'autant plus intéressante qu'elle ressemble au conte de fées traditionnel visant à décourager les adolescentes d'avoir des relations sexuelles... Malheur à celles qui se laissent séduire par l'inconnu, à savoir les hommes ${ }^{14}$.

Tombée malade, Marie-Ange assume passivement son destin de belle morte :

[... le père] amenait la malade aux récoltes. Au beau milieu du champ, tout l'été, on l'encanta dans des oreillers, parmi le foin jaloux de sa chevelure blonde. Ceux qui la voyaient, pure et belle, nimbée d'or comme les saints du Ciel, ne la nommaient autrement que l'Ange à Defroi. Avec l'ultime illusion de ceux qui vont mourir, elle rassurait chacun en disant : ça sera rien. (p. 71)

Celle qui fut désobéissante dans la vie s'avère parfaitement obéissante dans la mort. Qui plus est, la beauté

\footnotetext{
14 Il est intéressant de noter l'observation de David Décarie à l'effet que le « glouglou » venant de la bouche de la morte suggère l'hémorragie, « la mort la plus fréquente des filles qui se faisaient avorter ou qui tentaient elle-même l'opération » (2007, p. 79).
} 
éthérée de l'Ange à Defroi malade fait l'impasse sur sa corporalité et cette occultation a pour effet de nier la mort tout en permettant au père Defroi et au lecteur (de même qu'à l'écrivaine) de vivre une sorte de mort par procuration. Aussi la mort matérielle est-elle évitée puis supprimée. Une seule référence est faite dans le texte à la violence physique de la maladie de l'Ange à Defroi, à savoir le vomissement de sang (p. 71); l'accent est mis sur la patience de la malade. Mais comme l'illustre la présence insistante de la figure de la revenante chez Guèvremont, l'« ultime illusion », en fin de compte, n'est pas que la mort «[ne] sera rien», mais plutôt qu'elle est refoulée à jamais.

Le personnage de Caroline dans Tu seras journalisteœuvre largement autobiographique - aimerait que sa tentative de suicide relève du même système de représentation idéalisée ${ }^{15}$. Lorsqu'elle se rend à la morgue pour faire un reportage au sujet d'une inconnue qui s'est noyée, elle se rappelle la vision qu'elle s'était construite de son propre suicide un an auparavant :

Quand, à pareille date, l'an dernier, elle avait tenté de se suicider, la pensée que son corps serait transporté à la morgue ne l'avait pas même effleurée. Elle s'était vue en belle morte dans de la soie capitonnée, avec des fleurs de jardin tout autour d'elle. (TSJ, vol. 3, no 3, p. 22)

La matérialité du cadavre de l'inconnue vient troubler, cependant, cette image : «n[u] parmi les morceaux de glace », le cadavre est "violacé, marbré par plaques verdâtres, que l'enflure et la lueur des chandelles rendaient encore plus effrayant». La morte revient par la suite hanter Caroline:

15 Les références à cette œuvre seront désignées par le sigle TSJ. 
« Mais elle [Caroline] était déjà au-dehors, à la grande noirceur. Il lui semblait qu'elle ne posait pas le pied à terre, mais qu'une autre à sa place marchait dans de l'ouate irréelle, sans cependant parvenir à avancer. L'image de la morte la tirait en arrière » (TSJ, vol. 3 , no 3 , p. 22).

La scène de l'accouchement de Phonsine met en scène encore un autre spectre, qui apparaît, cette fois-ci, précisément au moment où le personnage féminin fait l'expérience de la réalité maternelle :

Phonsine n'avait pas dormi deux heures qu'elle s'éveilla net, comme si quelqu'un l'eût poussée à l'épaule. Le cœur battant, elle attendit. Peu à peu ses yeux s'habituèrent à l'obscurité, mais elle s'était trompée : il n'y avait personne dans la chambre. Malgré cela, convaincue d'une présence auprès d'elle, elle voulut s'asseoir dans son lit. (MD, p. 143)

Dans la « rouge vallée de la maternité », la femme morte attend.

Le passage du Survenant décrivant la fameuse inondation du Chenal du Moine renforce ce lien entre la maternité et la mort. Didace le patriarche raconte une histoire de survie masculine et de sacrifice maternel; Phonsine quant à elle, « écout[e] avidement» (p. 121) cette «belle histoire de [la] jeune femme de vingt ans, sainte et héroïque paysanne qui, sur le point d'accoucher, supplia son mari, au plus fort de l'inondation, de l'abandonner à la mort et de se sauver avec les deux autres enfants » (p. 121). En l'occurrence, la mère est vouée à mourir pour et de sa propre création, et il faut bien remarquer qu'il s'agit, une fois de plus, d'un destin mortifère qui serait inscrit d'emblée - dès le mariage - sur le plan onomastique. La mère martyre porte le nom de famille (voire du père) «Lavallée », appellation qui évoque la descente vers la 
mort ( "la vallée ») et l'absorption totale de la femme par celle-ci («l'avalée»). Ou, serait-il plus juste de parler non pas d'« absorption », mais de « résorption »? Car, il faut bien le noter, le féminin s'avale lui-même dans ce scénario : c'est la maternité qui tue, quoiqu'à l'aide d'un principe destructeur masculin (patriarcal). Enfin, le champ lexical de la résorption du féminin laisse entendre que le personnage «avide» de Phonsine éprouverait, inconsciemment, le désir de suivre l'exemple de cette mère légendaire lors de son propre accouchement («Des mains de feu [...] l'abandonnèrent, solitaire, dans la rougevallée de la maternité » MD, p. 144, je souligne).

Edgar Allan Poe affirme dans The Philosophy of Composition que la mort d'une belle femme est le plus poétique des sujets (p. 19). Alors que la description de la noyée de $T u$ seras journaliste met en évidence le côté matériel (voire grotesque) de la mort, la figure de la morte dans le cycle du Survenant appartient davantage à l'esthétique que décrit Poe, à un changement près qu'ici la qualité poétique de la figure est amplifiée lorsqu'il s'agit d'une bonne et belle femme. En témoignent le personnage esthétisé et vertueux de l'Ange à Defroi tout autant que la figure de la mère martyre; celle-ci renforce la relation entre la maternité et la spectralité féminine déjà présente dans l'histoire de celle-là.

Phonsine, la mère martyre de l'inondation, l'Ange à Defroi, le cadavre féminin inconnu à la morgue, et Caroline, la figure de l'écrivaine dans Tu seras journaliste, forment une généalogie fantomatique troublante, reliant l'écriture au féminin à la mort. À la lumière de cette étrange sororité spectrale, comment interpréter le cadavre féminin qui empêche Caroline d'«avancer» dans «la grande noirceur»? Peut-on 
mettre cette vision macabre en rapport avec la difficulté pour les femmes de cette génération de transition d'assumer pleinement un rôle d'écrivaine? La réaction de Caroline en apprenant qu'un autre a lu les lettres d'amour fictives qu'elle avait écrites - il s'agit du juge qui préside à son procès pour suicide - laisse entendre qu'écrire constitue une activité honteuse : «Comment avait-elle pu négliger de détruire toute cette littérature? On avait donc fouillé son passé jusque dans les moindres recoins » (TSJ, vol. 2, no 3, p. 28). De toute évidence, il vaut mieux ne pas laisser de traces littéraires. Force est de noter, d'ailleurs, que Caroline ne sera pas journaliste, le dénouement invraisemblable voulant qu'elle abandonne son rêve, retourne au village natal et se marie. Le journalisme, en fin de compte, n'aura été qu'un "grand vent d'illusion » (TSJ, vol. 3, nº 7, p. 24).

Les personnages féminins que peint Guèvremont forment en fait une longue série de doubles, la mort d'une femme se liant toujours à l'(in)existence d'une autre. Le cycle du Survenant s'avère ainsi l'histoire d'une revenance. Le lecteur du diptyque duSurvenant et de Marie-Didace a l'impression que la mère Beauchemin incarne la Femme à qui doivent se comparer toutes les autres, mais l'on apprend dans En pleine terre que Mathilde elle-même avait remplacé la grand-mère Beauchemin et qu'elle se sentait étrangère dans la maison Beauchemin (p. 33). Mathilde constitue donc la première survenante; Phonsine sera la suivante, en attendant l'arrivée de l'Acayenne. Chacune disparaîtra, à tour de rôle, derrière l'image de la figure maternelle précédente. Aussi les personnages féminins représentent-ils une variation de la même revenante : la femme morte est réincarnée pour être assassinée de nouveau. Dans toutes ces instances de gémellité, une femme s'efface : le double vient en effet anéantir la spécificité de celle 
qu'il remplace. Comment être unique, avoir l'impression d'exister en tant qu'individu, si l'on est interchangeable? Qui plus est, en se substituant à l'autre, le double se mêle au meurtre de celui-ci.

Ainsi, la femme singulière (tout autant que la femme réelle) disparaît derrière une série d'images, et avec chaque nouveau reflet, la figure de la femme morte s'amplifie. L'Acayenne, la dernière femme dans la série funèbre de redoublements et de revenances, incarne merveilleusement cette figure. " D'abord, elle est veuve », remarque le Survenant à son sujet $(\mathrm{LS}, 188)$. Il s'agit de la première description de celleci, qui la lie d'emblée à la mort. Lorsqu'elle périt à la fin de Marie-Didace, l'Acayenne réalise donc pleinement son destin, dans une splendeur rappelant celle de l'Ange à Defroi :

[...] l'Acayenne, allongée, droite sous les couvertures, paraissait reposer, magnifique dans sa chair. Sur son visage calme et légèrement penché, comme dans un moment de réflexion, la bouche gardait le pli du sourire. Une teinte rosée persistait aux joues et à la gorge. Des frissons, qu'une dernière sueur avait dû provoquer, ornaient le front lisse d'une frange d'or fané. Aucune trace d'agonie. (p. 213)

Curieusement, l'Acayenne est aussi «vivante » dans la mort que dans la vie.

\section{Conclusion}

Guèvremont a exprimé dans une de ses lettres à DesRochers «la peur folle de toutes [ses] extases; de ne pas [se] rendre jusqu'au bout, de n'en pas connaître la fin » (Lepage, 1990, p. 70-71); c'était à l'époque où elle était en train d'écrire la scène de la mort du père Didace. En effet, comme le pense celui- 
ci en apprenant la mort de son mentor de chasse Péloquin, on voit, dans Marie-Didace, le « commencement de la fin, un signe des temps » (p. 107). Ce "signe des temps » s'annonçait déjà, cependant, dans En pleine terre avec la noyade d'Ephrem, le fils vaillant qui aurait pu prolonger la tradition paternelle. Sa mort occasionne aussi bien le premier redoublement féminin que la première apparition spectrale du cycle. Tout d'abord, la mère Beauchemin, effondrée, se dit qu'elle devra "se reconstruire une autre personne » afin d'apprendre à vivre sans son fils (p. 53). Le passage qui suit met en scène le spectre : «Quelqu'un s'occupa de fermer les contrevents hormis ceux de la cuisine. Mais le malheur était quand même entré dans la maison » (p. 53). La hantise, en l'occurrence, annonce une rupture idéologique : le double féminin et le spectre font leur apparition au moment précis où les anciennes traditions commencent à s'écrouler.

Les quatre textes de Guèvremont explorés ici témoignent de deux désirs conflictuels : celui de renforcer le vieux système patriarcal en le représentant, et celui de provoquer son effondrement. Métaphore saisissante, la madone décapitée que voit Caroline lorsqu'elle fait un reportage sur un incendie souligne de façon radicale la difficulté — voire l'impossibilité — de réconcilier l'écriture et le féminin : «Après avoir recueilli toutes les notes qu'il lui fallait, Caroline se retourna pour un dernier regard. Entre deux glaçons, elle aperçut une madone sans tête qui pressait encore son Enfant-Jésus » (TSJ, vol. 2, no 12, p. 7). En jetant « un dernier regard» sur le passé et la tradition patriarcale, le personnage de l'écrivaine s'efforce de conjuguer son travail intellectuel et son rôle de femme. Le résultat en est une image violente qui se réfracte entre deux modes de représentation : l'ancien, et celui qui vient de naître. 


\section{Bibliographie}

BAtTERSBY, Christine (1989), Gender and Genius: Towards a Feminist Aesthetics, London, The Women's Press.

Bordo, SuSAN (1987), The Flight to Objectivity: Essays on Cartesianism and Culture, Albany, State University of New York Press, coll. «SUNY series in philosophy ».

BOUTHILlETTE, Jean (1959), « Germaine Guèvremont. Elle a écrit pour échapper à la peur », Photo-journal, 17-24 janvier 1959, p. 7.

BRONFEN, Elisabeth (1992), Over Her Dead Body: Death, femininity and the aesthetic, Manchester, Manchester UP.

DÉCARIE, DAVID (2001), "Le relais des survenants », Voix et Images, vol. 26, no 2, p. 359-83.

- (2006), «Résonances. L'interfiguralité chez Germaine Guèvremont », dans Annette Hayward (dir.), La rhétorique au féminin, Québec, Nota bene, p. 315-33.

—. (2007), « Le secret de Manouche : le thème de la fille-mère dans le Cycle du Survenant», Canadian Literature/ Littérature canadienne, vol. 195, p. 68-83.

GILBERT, SuSAN et SUSAN GUBAR (1979), The Madwoman in the Attic: The Woman Writer and the Nineteenth-Century Literary Imagination, New Haven, Yale University Press.

Grosz, ElizABeth (1994), Volatile Bodies: Toward a Corporeal Feminism, Bloomington et Indianapolis, Indiana University Press, coll. « Theories of Representation and Difference ».

GuÈVREMONT, GERMAINE (1939-1940), « Tu seras journaliste », Paysana, vol. 2, no 2, p. 12-13; vol. 2, no 3, p. 28-29; vol. 2, no 4 , p. 13 et 20; vol. 2, no 5 , p. 7 et 11; vol. 2, no 6, p. 10-11; 
vol. 2, no 7, p. 8-9; vol. 2, no 8, p. 6-7; vol. 2, no 9, p. 6-7 et 13; vol. 2, no 11 , p. 5-7; vol. 2, no 12 , p. 6-7; vol. 3, no 1, p. 18-19; vol. 3, no 1 (bis), p. 22-23; vol. 3, no 2, p. 24-25 et 32; vol. 3, no 3, p. 20-22; vol. 3, no 4, p. 9 et 24 ; vol. 3 , no 5, p. 19 et 24 ; vol. 3, no 6, p. 20-21; vol. 3, no 7, p. 22-24.

—. (1976 [1942]), En pleine terre, Montréal, Fides, coll. « du Goéland ».

—. (1990 [1945]), Le Survenant, Montréal, Bibliothèque québécoise.

—. (1992 [1947]), Marie-Didace, Montréal, Bibliothèque québécoise.

LECLERC, Rita (1963), Germaine Guèvremont, Montréal, Fides.

LEPAGE, Yvan (1990), "Cher Survenant...: Germaine Guèvremont - Alfred Desrochers (1942-1951)», Voix et Images, vol. 16, n 1, p. 64-78.

PoE, Edgar Allan (1984 [1846]), «The Philosophy of Composition », Essays and Reviews, New York, Library of America, coll. «Literary Classics of the United States », p. 1325.

SAINT-MARTIN, LORI (1997), «Sexe, pouvoir et dialogue », Études françaises, vol. 33, n 3 , p. 37-52.

SMART, PATRICIA (1988), Écrire dans la maison du père: L'émergence du féminin dans la tradition littéraire du Québec, Montréal, Québec/Amérique, coll. «Littérature d'Amérique ».

—. (1997), " "Changer la vie" ou "changer le monde" », Études françaises, vol. 33, no 3, p. 15-21. 


\title{
Résumé
}

Cet article propose une lecture du cycle du Survenant et du roman-feuilleton Tu seras journaliste de Germaine Guèvremont qui met en relief les signes d'une ambivalence envers l'acte d'écrire en tant que femme. L'aversion qu'éprouve le personnage de Phonsine à l'égard de sa grossesse, le redoublement de certaines figures féminines, et le retour spectral de la femme morte dans l'œuvre de Guèvremont sont les constituants qui appuient cette hypothèse.

\begin{abstract}
This article highlights the signs of ambivalence towards the act of writing as a woman that are present in Germaine Guèvremont's Survenant series, as well as her novella Tu seras journaliste. The aversion felt by the character Phonsine with regards to her own pregnancy, the doubling of certain female figures, and the spectral "return" of the dead woman in Guèvremont's work support this hypothesis.
\end{abstract}

